\title{
Incidencia del software multimedia EMILY'S y su impacto en la educación inicial
}

\section{Incidence of EMILY'S multimedia software and its impact on early childhood education}

\author{
Nelson Eduardo Mariscal Huacón ${ }^{1, *}$, Gladys Patricia Guevara Albán ${ }^{1,2, \dagger}$, y Cristian Salomón Guevara Albán ${ }^{1, \ddagger}$ \\ ${ }^{1}$ Instituto Tecnológico Superior Babahoyo, Ecuador. \\ \{nmariscal,gguevara,cguevara\}@institutobabahoyo.edu.ec \\ ${ }^{2}$ Universidad Técnica de Babahoyo, Ecuador. \\ gguevara@utb.edu.ec
}

Fecha de recepción: 22 de julio de 2016 — Fecha de aceptación: 18 de agosto de 2016

\begin{abstract}
Resumen-El software multimedia para la enseñanza de las relaciones lógico-matemáticas en la educación inicial hoy en día ha logrado cambios significativos en la docencia, dejando de utilizar métodos y técnicas tradicionales de enseñanza, para aplicar las tecnologías de la información y comunicación - TIC's dentro del proceso de enseñanza aprendizaje, y es ahí donde surge la imperiosa necesidad del uso de un software multimedia utilizándolo como eje transversal para la educación inicial. El objetivo principal de esta investigación fue aplicar un software multimedia que permita mejorar el proceso de enseñanza-aprendizaje en las relaciones lógico - matemáticas de los niños y niñas educación inicial del Centro de Educación Inicial "El Mamey". Cabe indicar que los factores que están influyendo en la enseñanza de los niños y niñas en edades comprendidas entre 3 y 5 años son: los hábitos, la motivación, la metodología, la familia y la inteligencia emocional, todo esto, conlleva al uso de un software multimedia que permita mejorar habilidades cognitivas, psicomotrices y más aún cuando se aprende jugando. Lo antes mencionado se basa en las encuestas realizadas a los padres de familia i/o representantes legales de los niños y niñas de educación inicial, donde se consideran factible el uso del software multimedia Emily's.
\end{abstract}

Palabras Clave-Software multimedia, relaciones lógico-matemáticas, educación inicial.

Abstract-The multimedia software for teaching logical-mathematical relationships in early childhood education today has achieved significant changes in teaching, leaving using traditional methods and teaching techniques to apply information technology and communications - ICT within the teaching-learning process, and that is where the imperative of using a multimedia software using it as a central focus for early childhood education arises. The main objective of this research was to apply a multimedia software to improve the teaching-learning process in logical relationships - math children early education Early Education Center . ${ }^{\text {El }}$ Mamey". It is noted that the factors influencing the teaching of children aged between 3 and 5 years are: habits, motivation, methodology, family and emotional intelligence, all this leading to use of a multimedia software to improve cognitive, psychomotor and even more so when you learn playing skills. The above is based on surveys of parents i / or legal representatives of children of early childhood education, where the use of multimedia software Emily's are considered feasible.

Keywords-Multimedia software, logical- mathematical relationships, childhood education.

\section{INTRODUCCIÓN}

$\mathbf{L}$ a comunidad educativa del Centro de Educación Inicial "EL MAMEY", considera que en esta nueva era de revolución tecnológica se debe estar en constante transformación y actualización digital, ya que en los salones de clases se muestra el desinterés de los estudiantes por el aprendizaje en las diferentes áreas y exclusivamente en la de relaciones lógico - matemáticas, es por esta razón, que a nivel mundial el desarrollo tecnológico se ha puesto al servicio de las instituciones educativas de todos los niveles y ha ido creciendo constantemente surgiendo la necesidad de hacer uso de ellas para incluirlas en las labores cotidianas de los actores principales de la educación que son los estudiantes.

\footnotetext{
*Ingeniero en Sistemas e Informática, Magister en Educación Informática.

${ }^{\dagger}$ Licenciada en Ciencias de la Educación en la Especialización de Informática Educativa, Magister en Informática Educativa y Multimedios Mención Desarrollo de Multimedios.

${ }^{\ddagger}$ Ingeniero en Sistemas.
}

La Constitución de la República del Ecuador, en sus artículos 26, 27, 28 señala que la educación es un derecho de las personas, un área prioritaria de la política pública e inversión Estatal, garantía de la igualdad e inclusión social y condición indispensable para el buen vivir. Estos aspectos mencionados añoran la importancia de brindar una calidad educativa que incorpore la innovación tecnológica que se va diseñando cada día con mejores opciones para el estudiante.

El deficiente desarrollo de las relaciones lógico-matemáticas es una situación que hoy en día preocupa a los docentes, ya que existe el desinterés, miedo y reacción adversa al estudiarla, lo que limita el desarrollo de nociones básicas a los niños y niñas en la educación. Es menester indicar que esto influye en los procesos de enseñanza-aprendizaje desplegado dentro y fuera de la institución educativa. El grado de preparación de los docentes es imprescindible para poder utilizar las nuevas tecnologías. Esta investigación se enmarca en la psicología cognitiva, la pedagogía, y las TIC's como herramientas de 
ayuda en la educación inicial actual.

Las nuevas tecnologías deben ser un recurso al servicio de la educación, es así que se han convertido en herramientas de trabajo donde el docente debe aplicarlos en su quehacer educativo, y así lograr, un aprendizaje óptimo en base a las opciones múltiples que permite el uso de un software multimedia. Además, con la aplicación de un software multimedia en la educación inicial actual permitirá cumplir un rol muy importante, que sin duda alguna hace que los niños y niñas se sientan motivados en conocer más sobre las relaciones lógico - matemáticas.

El MEC (Ministerio de Educación y Cultura) principal responsable de la educación nacional y comprometido con la necesidad de ofertar una educación de calidad que brinde igualdad de oportunidades a todos, pone a disposición de los docentes y otros actores de la Educación Inicial el currículo que permitirá guiar los procesos de enseñanza y aprendizaje en este nivel educativo.

El documento menciona la trayectoria curricular acumulada a este nivel educativo en el país, así como también, experiencias e investigaciones innovadoras sobre la primera infancia o educación inicial que se han elaborado dentro y fuera del Ecuador. Estos estudios constituyen el sustento técnico para el Currículo de Educación Inicial.

El Currículo de Educación Inicial surge y se basa en el derecho a la educación, atendiendo a la diversidad personal, social y cultural, además, identifica con criterios de secuencialidad, los aprendizajes básicos de este nivel educativo, adecuadamente articulados con el primer grado de la Educación General Básica. Además, contiene orientaciones metodológicas y de evaluación cualitativa, que guiarán a los docentes en el proceso de formación.

Relaciones lógico - matemáticas comprenden el desarrollo de los procesos cognitivos con los que el niño explora y comprende su entorno y actúa sobre él para potenciar los diferentes aspectos del pensamiento. Este ámbito debe permitir que los niños adquieran nociones básicas de tiempo, cantidad, espacio, textura, forma, tamaño y color, por medio de la interacción con los elementos del entorno y de experiencias que le permitan la construcción de nociones y relaciones para utilizarlas en la resolución de problemas y en la búsqueda permanente de nuevos aprendizajes (Educación, 2014).

Es imprescindible que los niños y niñas sean los actores principales de la educación inicial, de esta manera, poder inducirlos al desarrollo de habilidades que son tan necesarias para su crecimiento intelectual. El pensamiento lógico-matemático es construido por el niño desde su interior a partir de la interacción con el entorno. La asociación de operaciones mediante la clasificación, seriación e inclusión, posibilitan la movilidad y reversibilidad del pensamiento, necesarias en la construcción del concepto de "número". Este proceso constructivo comienza mucho antes del ingreso a la escuela. En palabras de Vigotsky, todo aprendizaje escolar tiene su historia previa. Por lo tanto, el niño o niña en su interacción con el entorno ha construido en forma "natural" nociones y estructuras cognitivas que deben continuarse desarrollando mediante la enseñanza escolarizada.

El investigador buscó estudiar cómo un material educativo multimedia basado en el método de lectura Doman facilitaría la enseñanza de la lectura en nivel preescolar, desde la perspectiva de los docentes. Para lograr esto, se buscó determinar los factores relacionados con el docente y la institución que influyen en la competencia lectora en el nivel preescolar, además, de diseñar y producir un material educativo multimedia fundamentado en la metodología Doman para la enseñanza de la lectura en preescolar. El prototipo diseñado fue Baby reader: método interactivo de lectura para edades tempranas, evaluado por docentes de escuelas preescolar privada y pública. Los docentes aportaron su opinión sobre los factores que influyen en la enseñanza de la lectura en preescolar, además, realizaron una evaluación al prototipo, considerando que es factible su uso y aplicación en un contexto institucional, facilita la enseñanza de la lectura y el docente cambia de su rol a mediador del aprendizaje al contar con el apoyo de la tecnología aplicada a la educación. Finalmente, se sugiere incorporar tecnologías al aula mediante la mejora de la infraestructura, el equipamiento y la capacitación.

Doman manifiesta que lectura es una de las funciones cerebrales más importantes del ser humano, indispensable para el desarrollo neurológico del infante, como afirma Goodman (2006) al señalar que la función antecede a la forma. La lectura es un proceso que debe enseñarse en casa por los padres, es tan importante como enseñar a caminar y no debería ser pospuesto hasta el ingreso a la escuela. En este sentido, no se podría hablar del método de lectura Doman aplicado a la escuela, ya que éste es un estilo de vida para los padres que desean educar a sus hijos en casa, creando las oportunidades necesarias para su desarrollo (Ángeles Valadez, 2013).

Los autores antes mencionados indican ¿De qué manera el diseño y producción de un material educativo multimedia basado en el método de lectura Doman, facilitaría la enseñanza de la lectura en el nivel preescolar desde la perspectiva de los docentes?, y por esta razón se determina que los factores relacionados con el docente y la institución influyen en el desarrollo de la competencia lectora en el nivel de preescolar y así mismo, diseñar, producir un material Educativo Multimedia bajo la metodología Doman, en formato DVD, para facilitar la enseñanza de la lectura en el nivel de preescolar. En relación al análisis de los datos recabados, y cumpliendo el primer objetivo.

La mayoría de las instituciones no tienen un espacio destinado para el conocimiento y apropiación de las tecnologías por parte de los infantes y los docentes que las incorporan, las utiliza únicamente para proyectar videos o escuchar música, lo que refuerza el planteamiento de Garassini (2005), que menciona que aunque la tecnología se ha venido incorporando en la educación preescolar, ésta carece en la mayoría de los casos de sustento pedagógico y metodológico.

El multimedia facilita y potencia la difusión, la información, enriquece la comunicación; admite la utilización de elementos lúdicos y recreativos, a la vez que individualiza la trasmisión del contenido (Trujillo, 1994).

\section{DesarRollo}

\section{Materiales y Métodos}

El tipo de investigación utilizado fue de campo, se realizó en la ciudad de Babahoyo en el Centro de Educación Inicial 
"El Mamey" que es una institución pública, donde se prestaron todas las facilidades, el apoyo de los compañeros maestros y maestras, de los estudiantes, padres y madres de familia para aplicarles una encuesta. Los instrumentos fueron aplicados a tres docentes participantes del nivel de educación inicial subnivel 1 y 2 , noventa padres de familia y también se realizó una ficha valorativa a los niños y niñas, cuyos resultados fueron divididos en dos momentos: el primero se refiere a los docentes y su metodología de enseñanza, y el segundo se refiere a los padres de familia como apoyo en la educación para los niños y niñas.

\section{Padres de familia como apoyo en la educación para los niños $y$ niñas.}

El $57 \%$ de padres de familia manifiestan que sus representados tienen problemas al identificar objetos y el $33 \%$ no. Un $55 \%$ de padres de familia afirman que sus hijos deben utilizar el ordenador y un $25 \%$ no, el $45 \%$ de padres de familia están muy de acuerdo manejar un software multimedia, un $78 \%$ están muy de acuerdo en manejar un ordenador.

\section{Ficha De Observación}

Los niños y niñas comprendidos entre los 4 y 5 años de edad del Centro de Educación Inicial carecen de nociones básicas al momento de interactuar en su entorno natural y cultural, esto se reflejó en la ficha valorativa que muestra como resultado que en la mayoría no identifican objetos, no reconocen los números, no distinguen el tiempo y espacio y otros factores que son muy influyente en su crecimiento intelectual.

\section{RESULTADOS Y DiscuSióN}

Al análisis de las encuestas realizadas a los padres de familia y representante legal podemos indicar, que la mayoría, manifiestan que sus representados tienen problemas al momento de identificar elementos, texturas, colores, desconocen de las nociones del tiempo y otros aspectos que se vuelven relevantes al momento de forjar a los estudiantes. Así mismo, se pudo evidenciar en base a la investigación de campo, no existe el correcto uso de la infraestructura tecnológica, que fue corroborado en las encuestas realizadas a los docentes y padres de familia, no sin antes mencionar que en la ficha valorativa aplicada a los estudiantes se pudo detectar la necesidad del uso de software multimedia para ayudar en su generación de conocimientos. Un resultado sorpresivo fue el relacionado con los profesores no dominan el uso del computador, sin embargo, todos ellos han tomado un curso de actualización tecnológica y creen que es importante su uso dentro de la práctica docente. En lo que se refiere al software multimedia, el resultado del análisis muestra que este tipo de software permite que el usuario interactúe de mejor manera con las funcionalidades del mismo, siempre y cuando se aplique considerar una metodología de desarrollo adecuada. Los actores de la comunidad educativa comparten en que se debe mejorar la comunicación entre estos miembros de la comunidad por lo que consideran que es uno de los factores que inciden en el bajo rendimiento académico. Los maestros coinciden en que los padres de familia deben colaborar en el control, seguimiento y prestar mayor atención a sus representados para ayudar en el desarrollo de sus capacidades.

\section{Conclusiones}

A través de la presente investigación se ha venido observando que las estrategias didácticas y tecnológicas no se están utilizando en la institución, debido la escasa infraestructura, no existen ambientes de aprendizajes educativos propicios que motiven, faciliten la adquisición de los conocimientos, el desarrollo de las habilidades y destrezas que permitan a los y las estudiantes a ser competitivos de acuerdo a sus necesidades, del contexto, la sociedad y comunidad educativa en general.

Que los software multimedia son importante para mejorar los procesos de enseñanza - aprendizaje, porque ayudan a motivar e interactuar con los niños y niñas, compañeros docentes, la integración de los padres de familia, mejorar la comunicación entre los diversos miembros de la comunidad educativa, además es una herramientas que el docente y educando pueden utilizar para realizar sus actividades de nivel curricular y extracurricular, y de esta manera, mejorar los conocimientos la familia Infantina, enmarcado por lo que sustenta el Ministerio de Educación en base a los estándares de calidad educativa.

La importancia y el uso adecuado que los miembros de la comunidad educativa El Mamey le den al software multimedia en base a la aceptación y positivismo a la propuesta realizada, sin ninguna duda nos permitirán alcanzar los objetivos plasmado en esta investigación.

\section{REFERENCIAS BIBLIOGRÁFICAS}

Ángeles Valadez, Miguel Ángel y Gómez Zermeño, M. G. y. G. M. I. A. (2013). Diseño de un recurso educativo multimedia basado en la metodología doman para mejorar la enseñanza de la lectura en el nivel preescolar.

Educación, M. d. (2014). Currículo de educación inicial.

Garassini, M. E. (2005). Incorporación de la informática en la Educación Inicial en Venezuela. Universidad de Los Andes (ULA).

Goodman, K. S. y. o. (2006). Sobre la lectura: una mirada de sentido común a la naturaleza del lenguaje y la ciencia de la lectura. Number Sirsi) i9789688536568.

Trujillo, Diego Aguilar y Morón, A. (1994). Multimedia en educación. Comunicar: Revista científica iberoamericana de comunicación y educación, (3):81-89. 